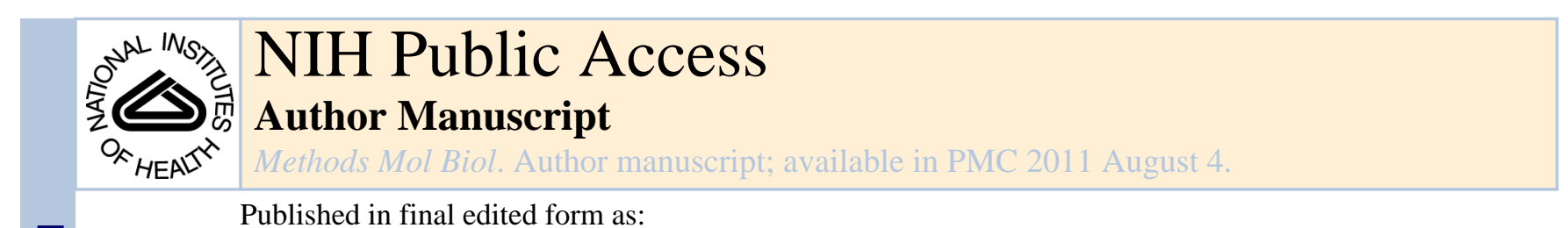

Published in final edited form as:

Methods Mol Biol. 2011 ; 770: 401-422. doi:10.1007/978-1-61779-210-6_15.

\title{
Ciona Genetics
}

\author{
Michael T. Veeman, Shota Chiba, and William C. Smith ${ }^{*}$ \\ Dept. of Molecular, Cell and Developmental Biology, University of California Santa Barbara, \\ Santa Barbara, CA 93106, USA
}

\begin{abstract}
Ascidians, such as Ciona, are invertebrate chordates with simple embryonic body plans and small, relatively non-redundant genomes. Ciona genetics is in its infancy compared to many other model systems, but it provides a powerful method for studying this important vertebrate outgroup. Here we give basic methods for genetic analysis of Ciona, including protocols for controlled crosses both by natural spawning and by the surgical isolation of gametes; the identification and propagation of mutant lines; and strategies for positional cloning.
\end{abstract}

\section{Keywords}

Ciona; ascidian; genetics; invertebrate chordate

\section{Introduction}

Ascidians are the closest invertebrate relatives of the vertebrates [1]. Their simple though stereotypically chordate larval body plans and their small, relatively non-redundant genomes have made ascidians an increasingly popular model system for developmental and systems biology [2-4]. With hermaphroditic reproduction and extremely polymorphic genomes, they are also well-suited for traditional forward genetics.

Although researchers make use of a wide range of ascidian species, here we will emphasize methods for Ciona intestinalis and Ciona savignyi, the two sequenced ascidians. Both have genomes of approximately $180 \mathrm{MB}$, which is roughly $1 / 20$ the size of most mammalian genomes, and $\sim 15000$ predicted genes, versus $\sim 23000$ in humans [5-8]. More importantly, many developmentally important gene families show considerably less redundancy than in vertebrate genomes [9-12]. This is thought to be due to both the ascidian lineage having diverged prior to two rounds of genome duplication in the vertebrate lineage [13], and also to extensive gene loss in the ascidians [14]. This decreased redundancy is extremely valuable for genetic analysis, and gives rise to a different spectrum of mutant phenotypes [15-17].

There are two unusual aspects of Ciona genetics that are important to keep in mind. The first is that Ciona are hermaphrodites that can be successfully self-crossed, which simplifies many aspects of genetic analysis. The second is that Ciona are astonishingly polymorphic, which has both advantages and disadvantages for the geneticist.

*corresponding author: w_smith@lifesci.ucsb.edu.

Notes 


\subsection{Self-fertility}

Ciona has been variously described as being both self-fertile and self-sterile. This is largely a matter of definition, and they are probably best described as being modestly self-fertile. Non-self fertilization is clearly favored [18], and a genetic mechanism for this preference has been determined [19]. Self-fertilization rates are extremely variable, ranging from $100 \%$ in some spawnings to $0 \%$ in others [20]. It is quite common for only $5-10 \%$ of eggs to selffertilize, which might be described as largely self-sterile by a stringent definition. However, in a typical clutch of 500 eggs, $5 \%$ self-fertilization gives 25 offspring. This degree of selffertility thus gives more than enough sampling power to reliably detect recessive mutations.

\subsection{Polymorphism}

In comparison to typical vertebrate genomes, ascidian genomes are extremely polymorphic. The two haplotypes derived from sequencing the genome of a single $C$. savignyi adult show a genomewide average Single Nucleotide Polymorphism (SNP) heterozygosity of $4.5 \%$ [21]. C. intestinalis is somewhat less polymorphic at 1.2\%-1.5\% [22]. By comparison, the human and chimpanzee genomes differ by less than $1.5 \%$, and humans differ from gibbons and siamangs by less than $5 \%$. This high polymorphism rate can be advantageous in that almost any arbitrary piece of amplified DNA is likely to contain a SNP or small indel and thus be usable as a genetic marker. This is particularly valuable given that efforts to construct inbred, isogenic lines have been unsuccessful to date. The high polymorphism rate is disadvantageous, however, in that it gives a high background when detecting mutations for positional cloning.

\subsection{Genomic Resources}

High-quality assemblies are available for both Ciona genomes [6,7,22]. Genomic scaffolds have also been placed onto linkage groups by FISH mapping in C. intestinalis [23] and by recombination mapping in $C$. savignyi [24]. There are large collections of ESTs, particularly in $C$. intestinalis [25,26], and a large database of $C$. intestinalis gene expression patterns by in-situ hybridization $[27,28]$. Good web resources include the Aniseed (http://aniseed-ibdm.univ-mrs.fr/) and Ghost (http://ghost.zool.kyoto-u.ac.jp/indexr1.html) databases and the Ensembl genome browser (http://www.ensembl.org).

\subsection{Genetic Strategies}

Although ascidians have been a model system for embryonic development for more than a century, they are a comparatively new model system for genetics. Ciona can be chemically mutagenized [20], and transposon-based mutagenesis is being actively developed [29]. Recessive mutations are sufficiently common in natural populations, however, that they can easily be identified by self-crosses of wild-caught individuals. Mapping strategies are evolving quickly, with earlier AFLP-based methods having been largely supplanted by direct mapping of SNPs. Methods based on deep sequencing are likely to be important in the near future. Here we emphasize the basic protocols for ascidian genetic analysis: controlled crosses; mutant screening; expanding mutant lines; DNA extraction; SNP mapping; and microinjection. For discussions of transient and stable trangenesis, refer to [29-31].

\section{Materials}

\subsection{Crosses}

1. Adult Ciona: Ciona intestinalis is widespread in temperate coastal waters [32], whereas C. savignyi is only found in Japan, the Pacific Coast of North America and the Atlantic Coast of Argentina [33]. Both are easily collected in marinas and harbors, particularly on pilings, buoys, and ropes that have been in the water for 
some time without having been cleaned. The undersides of unused dinghies and kayaks are also prime locations. There is considerable month-to-month variation in ascidian abundance at local marinas [34]. There are also seasonal variations, possibly reflecting changes in water temperature [35], with summer typically the best season in North America and Europe but the worst season in Japan. Be sure to follow local rules for collecting scientific specimens. Ciona are also available from the Ascidian Stock Center at UCSB (http://www.ascidiancenter.ucsb.edu), the Woods Hole Marine Biological Laboratory (http://www.mbl.edu/mrc/), the Roscoff Marine Station (http://www.sb-roscoff.fr/ModBiol/) and others.

Ciona are easily maintained in tanks or sea tables with running, unfiltered seawater. They can also be kept in recirculating systems with varying degrees of water exchange. Although not necessary in Southern California, some regions may benefit from heating or cooling mechanisms to stabilize water temperatures. Aquaculture system design is beyond the scope of this review and readers are directed elsewhere [20,36].

Be aware that $C$. intestinalis is divided into two partially reproductively-isolated subspecies, with Type A being found in the Pacific and the Mediterranean and Type B found in the Atlantic [32,37].

2. Plastic Cups: Disposable 16oz plastic cups (aka 'beer cups' or 'party cups') are ideal for spawning single Ciona (see Fig. 1A). They can be washed and reused many times. Label each cup near the brim to keep track of individual crosses.

3. Egg filters: Handy filters for collecting and washing eggs and embryos can be made from $50 \mathrm{~mL}$ centrifuge tubes and $70 \mu \mathrm{M}$ nylon mesh. For each tube, cut off the bottom with a band saw and drill or cut a large hole in the cap. Stretch a square of mesh over the tube threads and hold it in place with the drilled cap (see Fig. 1B). It is convenient to have a large number of egg filters, so as to not have to wash them between cups when screening large numbers of animals.

4. Petri Dishes: Several sizes of disposable plastic petri dish are needed: $60 \times 15 \mathrm{~mm}$ for screening the embryos from a single adult; $100 \times 15 \mathrm{~mm}$ for larger crosses; and $150 \times 15 \mathrm{~mm}$ for settling larvae to grow until maturity.

5. Net Twine: Twine or string is needed to hang plates and condos in the culture system. Lightly tarred nylon fishing net twine holds knots well and does not decay in seawater (Memphis Net and Twine Co., Memphis TN).

6. Scintillation Vials: 1 dram $(3.7 \mathrm{~mL})$ glass vials filled with water and capped make good weights to keep outcross plates and condos submerged in the culture system.

7. Cable Ties: 4" plastic cable ties (zip-ties) are used to attach vials, nametags, etc. to plates and condos, and to close condos when initially occupied.

8. Condos: Small cages ('condos') are needed to house individual ascidians in the culturing system after they have been detached from their substrate (see Fig 1C). Perforated plastic sheet of the type sold at craft stores as a substrate for needlepoint is a good material to build these cages. It is inexpensive, has holes of the right size, and does not foul with algae. Squares of this material can be sewn together with synthetic yarn to make boxes with one hinged side. Zip-tie a scintillation vial filled with water to the bottom of the box to weight it down. Tie a loop of net twine to the lid to suspend it in the culture system. Condos can be labeled with small squares of plastic cut from disposable lab weigh boats. Write the relevant details on the plastic label with an indelible lab marker, punch a hole in the label with a paper punch and zip-tie it to the condo. When an animal is first housed in a condo, the lid needs to 
be zip-tied shut so that it doesn't fall out. After 1-2 weeks, the animal will have attached to the wall of the condo and the lid should be opened to allow better water circulation (Fig. 1D).

9. Sperm Freezing Solution: $25 \%$ ultrapure DMSO (from a sealed glass ampule, eg. Sigma D2650) in seawater.

10. Sperm Freezing Rack: A Styrofoam tube rack that will float on the surface of a liquid nitrogen bath is needed to freeze sperm at the appropriate cooling rate.

\subsection{Mapping}

1. PCR primer pairs for mapping: PCR primers designed against intergenic regions often fail to amplify due to polymorphism in the primer binding sites. Avoid this by designing primers that are anchored in exon sequences. Keep primer melting temperatures similar, so that all markers can be amplified in the same run.

2. BSA-coated pipette tips: Fixed embryos and live dechorionated embryos are extremely sticky. All pipette tips used to transfer such embryos need to first be coated with a thin film of Bovine Serum Albumen (BSA) by briefly pipeting up and down a $10 \mathrm{mg} / \mathrm{ml}$ solution of BSA in seawater.

3. Agarose-coated dishes: Petri dishes to be used with fixed embryos and live, dechorionated embryos should first be agarose-coated. Make a melted 1\% agarose solution by heating. Fill one dish with the agarose and then immediately pour it from dish to dish, coating each one with a thin film. For live, dechorionated embryos, the agarose should be dissolved in seawater, whereas for DNA extractions from fixed embryos the agarose should be dissolved in deionized water.

\subsubsection{Single Tadpole/Small Pool DNA extraction for PCR}

1. $37 \%$ formaldehyde solution

2. $2 \times$ STL (Single Tadpole Lysis) buffer: $1 \%$ Triton-X 100, 100mM NaCl, 20mM Tris-HCl pH 7.8, $1 \mathrm{mM}$ EDTA.

3. $10 \mathrm{mg} / \mathrm{ml}$ Proteinase $\mathrm{K}$ in ultrapure water. Store in single-use aliquots at $-20^{\circ} \mathrm{C}$.

\subsubsection{Large-scale purification of genomic DNA}

Same materials as 2.2.3, but also including:

1. Disposable plastic pestles with matching $1.5 \mathrm{~mL}$ microcentrifuge tubes.

2. GuSCN buffer: $30 \mathrm{~g}$ guanidine thiocyanate, $2.5 \mathrm{~mL} 1 \mathrm{M}$ Tris- $\mathrm{HCl} \mathrm{pH} 6.4,2.2 \mathrm{~mL}$ 0.5M EDTA, 0.65g Triton-X 100, $25.8 \mathrm{~mL}$ ultrapure water.

3. Glass Milk (from GENECLEAN III kit, MP Biomedicals)

4. $70 \%$ Ethanol

5. Acetone

\subsection{Injections}

1. horizontal micropipette puller (eg. Sutter P-80)

2. $1 \mathrm{~mm}$ outer diameter, $100 \mathrm{~mm}$ long, thin wall borosilicate tubing with filling fiber (eg World Precision Instruments TW100F-4)

3. handmade holding pipette 
4. dissecting microscope (epifluorescence optics are helpful but not essential).

5. Coarse micromanipulator for holding pipette (eg. Narishige MN-151)

6. Fine oil hydraulic micromanipulator with 4 th axis axial advance for injection pipette (eg. Narishige MO-202U + MMO-220A)

7. PicoSpritzer pneumatic microinjector (Parker Hannifin Corp.) customized with a three-way vacuum-rated pneumatic toggle switch (eg. McMaster-Carr 8399K13). Connect the output from the switch to the input for the external solenoid valve. Connect one input to the regulated air pressure supply from the PicoSpritzer. Connect the other input to a source of regulated vacuum (eg. house vacuum with a regulator [McMaster-Carr 41585K41] and gauge [McMaster-Carr 3935K21]).

\section{Methods}

\subsection{Crosses}

Genetic crosses in Ciona can be performed by either dissecting out gametes and combining them in vitro, or by controlling the natural circadian spawning schedule by manipulating ambient light levels. Dissected crosses typically provide large numbers of extremely clean embryos (free from the fecal matter and pieces of sloughed tunic that are often filtered out with the eggs in a natural spawning), but have the disadvantage of killing the parents.

For both dissected and naturally spawned crosses, it can be helpful to keep the parental ascidians under constant light for 2-5 days to prevent spawning and thus allow the accumulation of gametes. A gravid animal will have a full oviduct (orange for $C$. savignyi, brownish-yellow for $C$. intestinalis) and a full sperm duct (white) clearly visible through the tunic.

\subsubsection{Collecting gametes by dissection}

1. Take an adult Ciona and gently remove the tunic by ripping it open with your fingers or slitting it with a small pair of scissors (see Fig. 2A,B). Take care not to rip or puncture the gonoducts.

2. Using fine forceps and a dissecting microscope, dissect through the muscle layer overlying the gonoducts (see Fig. 2C).

3. Use forceps to rip a small hole in the oviduct. Be careful not to rip the sperm duct yet.

4. Collect the eggs flowing out of the punctured oviduct with a Pasteur pipette (see Fig. 2D) and deposit them into an egg filter sitting in a cup of seawater.

5. Use closed forceps or the Pasteur pipette to gently stroke the oviduct towards the puncture site, forcing as many eggs as possible out of the hole. Collect them with the Pasteur pipette and move them to the egg filter.

6. Wash the collected eggs by transferring the egg filter through two cups of seawater. Eggs are best used immediately, but typically remain fertilizable for several hours. For critical experiments it is best to wait for 2 hours to ensure that the eggs were not inadvertently self-fertilized.

7. Puncture the sperm duct by ripping a small hole in with forceps.

8. Collect the sperm spilling out of the hole with a new Pasteur pipette. Try to collect the sperm as "dry" and clean as possible (see Fig. 2E). 
9. Transfer the sperm to a $1.5 \mathrm{~mL}$ microcentrifuge tube. If it is not to be used right away, store it at $4{ }^{\circ} \mathrm{C}$. Sperm can typically be stored at $4{ }^{\circ} \mathrm{C}$ for up to one week.

10. If you are dissecting multiple individuals, be sure to clean your forceps and use fresh Pasteur pipettes between each individual. An easy way to prevent sperm cross-contamination is to rinse your forceps off with a squirt bottle of seawater, dip them into a beaker of ethanol, and then rinse them again with the squirt bottle of seawater.

\subsubsection{In-vitro fertilization}

1. Place the eggs to be fertilized in an egg filter sitting in a cup half full of seawater.

2. Add a small amount of sperm. If the sperm was collected at full concentration, then 5 or $10 \mu 1$ is usually enough to fertilize thousands of eggs. Polyspermy is seldom a problem, however, unless the eggs have been dechorionated.

3. Swirl the cup gently until the sperm is dispersed.

4. Wait at least 10 minutes for fertilization to take place, then remove the filter from the cup, invert it over a $100 \mathrm{~mm}$ petri dish and gently rinse the eggs into the dish with a squirt bottle of seawater.

5. Incubate embryos at $18{ }^{\circ} \mathrm{C}$. The first cleavage should occur approximately one hour after fertilization, and can easily be observed with a dissecting scope (see Note 1).

\subsubsection{Natural Spawn}

1. Place the ascidians to be crossed in a cup, tub or bucket of seawater in a room or chamber with timer-controlled lighting (see Note 2).

2. C. savignyi: Set the timer to turn the lights OFF 6 hours before the desired time of spawning and then $\mathrm{ON}$ at the desired time of spawning.

C. intestinalis: Place the animals in the dark for 30 minutes.

3. As the lights come on, transfer the adults to a clean container of seawater. (This step is optional, but gives cleaner eggs with less fecal matter)

4. Spawning will typically occur roughly 15 minutes after the lights come $\mathrm{ON}$ for $C$. savignyi, and 50 minutes after the lights go OFF for $C$. intestinalis. Fertilization typically takes another 10 minutes, but may take as much as 60 minutes for a selfcross.

5. It is best to remove the adults after spawning to prevent them from eating their eggs.

6. At some point between fertilization and hatching, the eggs need to be moved from the spawning container to a petri dish so that they can be inspected under a dissecting microscope:

6a) Pour the water containing fertilized eggs/embryos through an egg filter.

6b) Invert the egg filter over a $100 \mathrm{~mm}$ petri dish and rinse the eggs/ embryos into it with a squirt bottle of seawater.

$118{ }^{\circ} \mathrm{C}$ is a good default temperature for culturing Ciona embryos, but for experimental convenience they can also be cultured as cool as $12{ }^{\circ} \mathrm{C}$ to slow down development.

${ }^{2}$ We normally perform natural spawnings in an environmental chamber kept at $17^{\circ} \mathrm{C}$. Room temperature is generally fine in a cool, air-conditioned room, but it is best to keep Ciona between $12{ }^{\circ} \mathrm{C}$ and $20^{\circ} \mathrm{C}$. 
7. Incubate embryos at $18{ }^{\circ} \mathrm{C}$. The first cleavage should occur approximately one hour after fertilization, and can easily be observed with a dissecting scope (see Note 1).

\subsubsection{Settling larvae onto Petri dishes}

1. At $18{ }^{\circ} \mathrm{C}$, hatching will occur approximately 17 hours after fertilization (see Note 1). Rinse the hatched larvae in an egg filter by sequentially placing the filter into 2 cups of fresh seawater. This removes dead sperm and fragments of chorion that may inhibit attachment.

2. Label a $150 \mathrm{~mm}$ petri dish on the outside rim (top and bottom) with the relevant genotype, date, etc.

3. Invert the filter over the LID of a large petri dish, placed concave side upwards, and rinse the hatched larvae into the lid with a squirt bottle of seawater. For a typical outcross using eggs from 4-6 wildtypes, split the larvae between $6-8$ sets of dishes.

4. Fill the lid with seawater until it is nearly but not quite overflowing.

5. Float the BOTTOM of the petri dish (concave side up) on top of the water in the lid (see Fig. 1F). This allows larvae to attach to both surfaces, and minimizes the air/ water interface where larvae often become trapped.

6. Attachment and metamorphosis are somewhat variable, but most larvae will have attached and metamorphosed after two days.

7. There are often some healthy larvae that have, for whatever reason, failed to attach. These can often be induced to attach by collecting them in an egg filter, inverting the filter over a dry petri dish at a height of 10-12 inches and rinsing them onto the dish with a squirt bottle of seawater.

8. Some mutants are competent to undergo metamorphosis but seldom do so because they cannot swim (eg. aimless [16]). These can sometimes be forced to attach by repeated dropping from a height or by manually forcing their palps against the dish with a hair loop or a fine pipette tip (eg. gel loading tip).

9. Plates should be moved to the culture tanks 2-5 days after plating (see Note 3 ).

Take each plate and make two holes on opposite sides of the rim with a hot soldering iron (best done in fume hood). Use a zip tie to attach a glass scintillation vial full of water to one hole. This acts as a weight to keep the plate submerged. Tie a loop of string through the other hole, to suspend the plate in the culture system (Fig. 1G). Attached juveniles can be seen as small dots on the plate when viewed at an angle (see Fig. 1H).

10. Juveniles are particularly susceptible to predation by nudibranchs. If your culture system uses raw seawater, be sure to check frequently and remove any nudibranchs or their eggs.

11. The generation time depends on the temperature, feeding levels and other environmental conditions, but sexual maturity typically takes 3-4 months (see Fig. 1I).

\footnotetext{
${ }^{3}$ There is considerable flexibility in when you move outcross plates to the culture facility. One can do so as soon as a sufficient number of larvae have attached to the plate, but it is sensible to wait for another two days or so to ensure that they undergo metamorphosis. It is best to move them within 5 days of metamorphosis, however, as they need to start eating. The water in the dish can be changed every $2-3$ days.
} 


\subsubsection{Screening for mutations in wild populations}

1. Collect 50 or more wild Ciona adults.

2. Leave them under constant light for several days to accumulate gametes.

3. Take 50 adults that have both sperm and eggs and array them into 50 labeled cups of seawater. (More or fewer can be screened, but 50 is a convenient number that fits onto the top shelf of standard lab cart and can easily be filtered in a few hours)

4. Induce self-fertilization by natural spawning as described in protocol 3.1.3.

5. Filter the embryos into labeled petri dishes. Be careful to ensure that the label on each dish of embryos matches the label of the cup holding the parent.

6. After hatching, examine each dish with a dissecting scope for a phenotype affecting $25 \%$ of the progeny (see Note 4 ) (see Fig. 1E).

7. It is likely that some animals will have failed to spawn, or will have spawned but the eggs will not have fertilized. If these animals still have gametes, they can be set up for another spawning cycle and retested (see Note 5). This can be done immediately for $C$. savignyi or after a 6 hour wait for $C$. intestinalis.

8. Potential mutant carriers should be housed in condos and moved to the culturing system for 1-2 weeks to recover.

9. After 1 or 2 weeks, respawn the putative carriers. This second screening is important to reduce false positives due to bad clutches of eggs or other nonMendelian causes of aberrant embryos (see Notes 6,7).

10. Putative carriers should be outcrossed (protocol 3.1.4) so as to expand the line and allow testing for Mendelian inheritance in the second generation, complementation testing against previous mutations, phenotypic analysis, sperm freezing, etc. (see Note 8). Offspring from self-crosses can also be settled onto plates, but outcrosses are typically much more efficient for expanding mutant lines, and sib-crosses are better than self-crosses for testing homozygote viability.

\subsubsection{Outcrossing a mutant}

1. Set up a known mutant carrier to spawn naturally.

2. Approximately 90 minutes before spawning, dissect eggs from several putatively wildtype Ciona keeping the eggs from each animal separate. Wait one hour and check that the eggs are not cleaving. If they are cleaving, discard that batch of eggs.

\footnotetext{
${ }^{4}$ Subtle phenotypes can be difficult to identify if the larvae are actively swimming. They can be anaesthetized by adding a few $\mathrm{mL}$ of $0.2 \%$ MS-222 (tricaine) to the dish.

${ }^{5}$ In our experience, it is extremely common for a second spawning to give fertilized eggs when the first spawning did not. This is potentially because both sperm and eggs are not always released with every light/dark cycle.

${ }^{6}$ Typical mutant phenotypes include short tails, club-shaped heads, and pigmentation defects. There are several types of phenotypes that we have found to rarely show Mendelian inheritance. These include tails with multiple severe kinks, and tails that are only slightly shorter than wildtype. These phenotypes typically present at either significantly less than or more than the expected 1:3 ratio, which is another sign that they are not Mendelian.

${ }^{7}$ An outcross can be performed simultaneously with the second screening by using filtered, sperm-containing water from the self-cross to fertilized wildtype eggs. This is sensible if the founder adult seems at all unhealthy or the putative mutation is particularly precious. If the second screening is negative, then the outcross can simply be discarded.

${ }^{8}$ It is always a quandary whether to keep the founder animal alive as long as possible for further experimentation or whether to kill it and freeze its sperm as a precaution against losing the line. This is left to the researcher's discretion, and should depend on the apparent relative robustness of the founder animal versus its outcrosses.
} 
3. Take a sample of eggs from each putatively wildtype animal and fertilize them with self sperm. This is a control to ensure that these animals are not themselves carrying any phenotypically meaningful recessive mutations.

4. When the animal starts spawning, put an egg filter (or multiple filters) containing unfertilized wildtype eggs into the container holding the known mutant carrier. As long as the top of the filter is kept above the water level, sperm can freely pass through the filter mesh to fertilize the wildtype eggs while keeping these eggs separate from the eggs spawned by the mutant adult. Alternatively, add spermcontaining seawater from the mutant (filtered through an egg filter) to dishes containing wildtype eggs.

5. After an hour, remove the egg filter and rinse the eggs into a petri dish. Check for cleavage with a dissecting scope.

6. After hatching, confirm that the control self-crosses do not show any mutant phenotype. If a phenotype is apparent, one option is to discard the outcross and try again. If the new phenotype is interesting, however, or if the outcross is particularly precious, then the outcross can be kept and screened for single and double heterozygotes.

7. After hatching, settle the outcross larvae onto petri dishes as described in protocol

\subsection{4.}

3.1.7 Identifying carriers-The simplest way to identify carriers from an outcross is to do self-crosses by spawning them individually in cups. This is reasonably effective, but there is always a variable fraction of animals that fail to self-fertilize. An efficient alternative is to add a small amount of sperm to each cup from a known carrier. This could be frozen sperm from a previous generation, fresh sperm from a previously identified carrier, or seawater in which a previously identified carrier(s) had just been naturally spawned and then strained through an egg filter to remove any eggs.

Alternatively, a small amount of sperm-containing seawater $(\sim 50 \mathrm{~mL})$ can be removed from each cup 25 minutes after the lights come on. This can then be mixed, strained through an egg filter to remove any eggs, and redistributed to each cup. This gives the eggs in each cup an opportunity to self-fertilize, and then a second opportunity to fertilize with sperm mixed from all of the potential carriers. As there should be 50\% carriers in a typical outcross, $25 \%$ of the sperm in the mixed solution should carry the mutation, giving $~ 1 / 8$ mutant progeny if an animal is a carrier but failed to self-fertilize (see Note 9).

3.1.8 Obtaining large numbers of mutant embryos-Phenotypic analyses and genetic mapping benefit from having large numbers of mutant embryos. Dissected crosses between multiple carriers work well, but will quickly reduce your numbers of identified carriers. The best way to get as many embryos as possible without killing any carriers is to do large group spawns. 5-10 gravid carriers can be placed in a tub or bucket and spawned en-masse, giving rise to thousands of mutant embryos. These large group spawns typically give very good fertilization rates.

An alternative method if you do not have large numbers of known carriers is to do bulk spawns of outcrosses before they have been identified. A whole plate of animals derived

\footnotetext{
${ }^{9}$ One issue with this method is that it may not reliably detect a second mutation inadvertently segregating in the animals being screened if it is present at much less than $25 \%$. Such mutations should be unusual, however, if the wildtypes used for outcrossing are confirmed to be truly wildtype.
} 
from an outcross can be spawned together, giving rise to extremely large numbers of offspring with $\sim 1 / 16$ being mutant.

\subsubsection{Freezing Sperm}

1. Aliquot $80 \mu \mathrm{L}$ sperm freezing solution into each of several $1.0 \mathrm{~mL}$ cryovials.

2. Collect as much clean, dry sperm as possible by dissection (protocol 3.1.1).

3. Add $20 \mu \mathrm{L}$ sperm to each tube, gently mixing the sperm with the sperm freezing solution.

4. Gradually freeze each tube in a Styrofoam rack floating on liquid nitrogen.

5. After 10 minutes, store the tubes in liquid nitrogen. (see Note 10)

\subsubsection{Thawing Sperm}

1. Remove 1 tube of frozen sperm from liquid nitrogen.

2. Quickly thaw the frozen sperm by pipetting $1 \mathrm{ml}$ of seawater up and down in the tube.

3. Quickly add the thawed sperm to unfertilized eggs.

\subsection{Linkage mapping and positional cloning}

The first ascidian mutations to be positionally cloned were mapped by AFLP analysis of bulked pools of phenotypically wildtype versus mutant tadpoles [16]. AFLP remains a viable option, but has largely been supplanted in our lab by mapping SNPs by direct sequencing. The Ciona genomes are sufficiently polymorphic that most $\sim 500 \mathrm{bp} \mathrm{PCR}$ products are expected to contain at least one SNP.

3.2.1 SNP mapping (see Figure 3)-Initial mapping is best performed on pools of DNA extracted from either mutant tadpoles (at least 20, with 50-100 being better) and an equivalent number of phenotypically wildtype siblings. Initial mapping should use embryos derived from a single selfed individual, so that no more than two alleles are present at any marker. The two pools of DNA are then used as template for a panel of PCR reactions using primer pairs designed to span the genome. We have had good results with a $C$. intestinalis panel consisting of two primer pairs evenly spaced on each chromosome arm, and with a $C$. savignyi panel with a primer pair near the center of each of the largest 96 reftigs. Direct sequencing of the PCR products can then be used to look for SNPs in the wildtype pool. If the marker is linked to the mutant locus, it will be visible as a change in the relative chromatogram peak heights for the two SNP alleles in the mutant pool versus the wildtype pool. In the case of tight linkage, only one peak will be visible. This is typically the smaller of the two alleles in the wildtype pool, as the SNP allele linked in cis with the mutant allele will be underrepresented in the wildtype pool.

A good metric for quantifying linkage in this assay is to take the ratio of the relative peak heights for the two pools $\left(\mathrm{H}_{\mathrm{mutA}} / \mathrm{H}_{\mathrm{mutB}}\right) /\left(\mathrm{H}_{\mathrm{wtA}} / \mathrm{H}_{\mathrm{wtB}}\right)$. This normalizes for contextdependent variation in incorporation frequency by the sequencing polymerase. This method is only semiquantitative, however, and potential weak linkage needs to be confirmed by testing other linked and unlinked markers.

\footnotetext{
${ }^{10}$ It is a good idea to test that the freezing was successful by thawing one aliquot and confirming that it can fertilize eggs.
} 
Once initial linkage to a chromosome arm or genomic scaffold has been determined, a finergrained panel of primer pairs should be designed against that region (keeping in mind that the mutant locus may still be quite far away...). This can be used to narrow the candidate region to a region of tight linkage flanked by markers showing appreciable recombination.

SNP mapping by direct sequencing of PCR products from large pools is ultimately limited by the inability to determine small differences in allele frequency as a function of chromatogram peak height (see Note 11). For finer scale mapping, it is necessary to switch to smaller pool sizes (we have been able to detect a single recombinant in a pool of five tadpoles) or else to perform single tadpole PCR (protocol 3.2.3).

3.2.2 Refining the candidate gene list-Once the critical interval has been narrowed by recombination mapping to $\sim 500 \mathrm{~kb}$, it becomes feasible to begin examining candidate genes (although if mutant tadpoles are plentiful, finer genetic mapping will always be the most unambiguous way of narrowing the field of candidates). A $500 \mathrm{~kb}$ interval typically contains $\sim 50$ genes. The genome browsers at Ensembl and Aniseed provide good starting points for inspecting the region and searching for obvious candidates.

The $C$. intestinalis EST and in situ collections are often helpful for tentatively narrowing the candidate list. These databases can also be helpful for $C$. savignyi after identifying the orthologous gene.

For all of the mutations we have cloned so far, the mutant gene transcript is absent or downregulated compared to wildtype [15-17]. This likely reflects a nonsense-mediated decay mechanism. Although not definitive, this is another important method for narrowing the candidate list.

Once a strong candidate is apparent, the coding regions should be amplified in overlapping segments from mutant genomic DNA and sequenced to look for mutations. Keep in mind that Ciona are extremely polymorphic, so a polymorphism that does not cause a severe predicted truncation or interfere with a known active site, etc., will need rigorous functional confirmation by rescue or knockdown.

\subsubsection{Single Tadpole/ Small Pool DNA Extraction for PCR}

1. Fix embryos by adding $1 / 10$ volume of $37 \%$ formaldehyde. 10 minutes of fixation is sufficient, and more is potentially harmful.

2. Wash the embryos twice with deionized water. The test cells, a maternally derived cell type on the outside of the embryo, should fall off (see Fig. 1J,K and Note 12). If they do not, move the embryos to an egg filter and rinse them with a stream of water from a squirt bottle.

3. The fixed embryos are sticky, so move them to agarose-coated dishes and use BSA-coated pipette tips.

\footnotetext{
${ }^{11}$ The ability to detect a single recombination event in pooled DNA is limited by noise in the chromatogram and by contaminating maternal DNA (see Note 12). If the chromatogram is extremely clean and there is no maternal contamination, then it is possible to detect a minor peak that is a tenth the size of the major peak (ie. a single recombinant in a pool of 5 embryos). That is probably the best case scenario, however, and smaller pools are more robust. Single tadpole PCR provides the most unambiguous results, but requires more sequencing to examine the same number of meioses.

12 There is a population of maternally-derived cells known as test cells that are found between the embryo and the chorion. After hatching, the test cells are found in the larval tunic on the outside of the larva (Fig 1J,K). Unless removed, the test cells are a source of contaminating maternal DNA that may confound genotyping assays. The test cells can be removed by dechorionating fertilized eggs, but dechorionation is a relatively harsh procedure that often gives a background of perturbed embryos. Alternatively, formaldehyde fixation has the counterintuitive property of loosening the test cells so that they can be washed off the embryo.
} 
4. The fixed embryos can be kept at $4{ }^{\circ} \mathrm{C}$ for several days. This can be helpful if large numbers of mutant and wildtype siblings need to be sorted.

5. Transfer single tadpoles to individual PCR tubes (or plate wells) in $5 \mu 1$ of water. For small pools, vary the volumes accordingly.

6. Add $5 \mu 1$ STL buffer and $1 \mu 1$ of $10 \mathrm{mg} / \mathrm{ml}$ proteinase $\mathrm{K}$.

7. Seal well and incubate for at least 12 hours at $55^{\circ} \mathrm{C}$.

8. Heat to $95{ }^{\circ} \mathrm{C}$ for 10 minutes to inactivate the proteinase. Spin down and store at $-20^{\circ} \mathrm{C}$.

9. $1 / 5$ of an embryo equivalent is usually sufficient for robust amplification.

3.2.4 Large-scale purification of genomic DNA-Although standard phenol/ chloroform preps can be used, we have obtained much better yields using a modified guanidinium/glass milk procedure:

1. Add $\sim 1000$ embryos (fixed or unfixed) to a $1.5 \mathrm{~mL}$ tube (use the tube that comes with a disposable plastic pestle.

2. Centrifuge at $400 \mathrm{~g}$ to pellet embryos and remove as much seawater as possible.

3. Add $200 \mu \mathrm{l} 1 \times$ STL buffer and $20 \mu 110 \mathrm{mg} / \mathrm{ml}$ proteinase K. Homogenize with disposable plastic pestle.

4. Incubate lysate at $55^{\circ} \mathrm{C}$ for at least 12 hours.

5. Add $600 \mu \mathrm{l} \mathrm{GuSCN}$ buffer and $10 \mu 1$ resuspended glass milk to the lysate.

6. Incubate ten minutes at room temperature, briefly vortexing every few minutes.

7. Centrifuge for 30 seconds at $12000 \mathrm{~g}$. Discard the supernatant to GuSCN waste.

8. Wash pellet once by adding $200 \mu \mathrm{l} \mathrm{GuSCN}$, resuspend by brief vortexing, spin down for 30 seconds at $12000 \mathrm{~g}$ and discard the supernatant.

9. Wash pellet twice as above with $200 \mu 170 \%$ ethanol.

10. Wash once with $200 \mu \mathrm{l}$ acetone.

11. Dry the pellet by leaving the tube lid open for $10-15$ minutes at $55^{\circ} \mathrm{C}$.

12. Add $20 \mu \mathrm{l}$ water. Resuspend and heat at $55^{\circ} \mathrm{C}$ for 10 minutes

13. Vortex and centrifuge for 5 minutes at $12000 \mathrm{~g}$.

14. Remove the supernatant to a new tube and store at $-20{ }^{\circ} \mathrm{C}$ (see Note 13).

\subsection{Injections}

Although not strictly a genetic method, microinjections can be invaluable in confirming candidate loci by rescue or morpholino knockdown.

Ciona eggs are small and have a tough, elastic cortex that can be difficult to pierce. Once pierced, however, the eggs are quite delicate, and lyse easily. $C$. savignyi eggs can be injected through the chorion, whereas $C$. intestinalis eggs have a tougher chorion and are best dechorionated before injection. Here we will focus on injecting $C$. savignyi eggs through the chorion.

\footnotetext{
${ }^{13}$ This prep copurifies large amounts of RNA with the DNA. If RNA-free DNA is required, treat the sample with RNAse and then ethanol precipitate.
} 
We pull needles from $1 \mathrm{~mm}$ thin wall borosilicate tubing with a filling fiber on a Sutter P-80 horizontal puller. Good needles need to be tested empirically for their ability to pierce the egg without lysing it. Fill needles from the back with a capillary loading pipette tip and let the injection solution wick to the front of the needle by capillary action. Once the injection pipette is mounted in the injection rig, it can be broken open by gently tapping it against the wall of a scratch made in the floor of the injection petri dish using a pair of fine forceps.

A holding pipette is used to keep the egg in place for microinjection. These are pulled by hand. First roll the center of a length of capillary tubing in a Bunsen burner flame. When it starts to soften, remove it from the flame and quickly pull it apart by $\sim 3 \mathrm{~cm}$. Cut the capillary in half, at the center of the thinned central section, by scoring and snapping. Take one half and repeatedly but briefly dip the thin end into the flame to constrict the tip. Check with a dissecting scope for the desired shape (see Fig. 4D). Lastly, form a hockey stick-shaped bend in the pipette by holding it briefly in the edge of the flame until it softens and droops.

We use a dissecting scope with epifluorescence optics mounted with two micromanipulators (see Fig. 4A,B). The left manipulator is a basic one that is only used to position the holding pipette. The right manipulator is a 4 axis oil hydraulic manipulator. The holding pipette is connected to a $10 \mathrm{~mL}$ syringe to manually provide a slight negative holding pressure. The injection pipette is connected to a custom modified pneumatic microinjector.

The main peculiarity about injecting Ciona eggs is that they have such a tough, elastic egg cortex that they cannot be injected by pressure alone, but require an initial pulse of vacuum to break the egg cortex. Without this pulse of vacuum, it can appear that you are injecting into the cytoplasm, but the injected material either remains confined to a membrane-bound vesicle or else leaks back out of the egg. Traditionally, both the positive and negative pressure were supplied manually with a syringe. We have obtained significantly better results using a pneumatic microinjector modified to provide pulses of vacuum as well as positive pressure.

The Picospritzer brand of microinjector uses a single external solenoid valve box that normally controls the flow of air between a pressure regulator and the needle holder. We added a toggle-controlled pneumatic switch allowing the source to the solenoid valve to be switched between the normal regulated positive pressure and a source of regulated vacuum. When the toggle is set to "Blow" the microinjector acts as a normal, pneumatic microinjector. When the toggle is set to "Suck", the microinjector delivers pulses of vacuum (see Fig. 4C).

To inject an egg, position the holding pipette in the middle of the field of view and move the petri dish around until an egg is near its tip. Pull back a little on the syringe connected to the holding pipette to capture the egg against the holding pipette. Use the XYZ joystick controls of the hydraulic manipulator to move the needle tip in line with the center of the egg (this will put the tip somewhat above and to the right of the egg). Use the axial control to slowly advance the needle tip through the chorion and into the center of the egg (see Fig. 4D). Set the toggle to 'Suck' and apply vacuum until a small amount of egg cytoplasm can be seen moving into the needle tip. Switch the toggle to 'Blow' and apply small pulses of positive pressure until the injected droplet is roughly $1 / 8$ the diameter of the egg. Use the axial advance to rapidly remove the needle from the egg. Push forward a little on the holding pipette syringe to release the injected egg. Move it to a new dish with a P20 micropipette.

Injections are typically performed with $0.2 \%$ Fast Green (which fluoresces in the red) or labeled dextran as a marker dye. Epifluorescence optics on the injection scope are helpful for confirming injection, but not strictly necessary. Fast Green fluorescence is typically not visible while it is being injected, but appears rapidly as it diffuses into the egg cytoplasm. 
Injection concentrations need to be titrated individually, but DNA is typically injected at 30$100 \mathrm{ng} / \mu \mathrm{l}$, RNA at $20-60 \mathrm{ng} / \mu \mathrm{l}$, and morpholinos at $0.05-0.2 \mathrm{mM}$. The injected volume can be estimated as a function of the radius of the injected droplet, but as this varies with the cube of the radius it is quite imprecise and should be thought of as only semi-quantitative.

\section{References}

1. Delsuc F, Brinkmann H, Chourrout D, Philippe H. Tunicates and not cephalochordates are the closest living relatives of vertebrates. Nature. 2006; 439:965-968. [PubMed: 16495997]

2. Imai KS, Levine M, Satoh N, Satou Y. Regulatory blueprint for a chordate embryo. Science. 2006; 312:1183-1187. [PubMed: 16728634]

3. Passamaneck YJ, Di Gregorio A. Ciona intestinalis: chordate development made simple. Dev Dyn. 2005; 233:1-19. [PubMed: 15765512]

4. Lemaire $P$, Smith WC, Nishida H. Ascidians and the plasticity of the chordate developmental program. Curr Biol. 2008; 18:R620-R631. [PubMed: 18644342]

5. Dehal P, Satou Y, Campbell RK, Chapman J, Degnan B, De Tomaso A, Davidson B, Di Gregorio A, Gelpke M, Goodstein DM, et al. The draft genome of Ciona intestinalis: insights into chordate and vertebrate origins. Science. 2002; 298:2157-2167. [PubMed: 12481130]

6. Satou Y, Mineta K, Ogasawara M, Sasakura Y, Shoguchi E, Ueno K, Yamada L, Matsumoto J, Wasserscheid J, Dewar K, et al. Improved genome assembly and evidence-based global gene model set for the chordate Ciona intestinalis: new insight into intron and operon populations. Genome Biol. 2008; 9:R152. [PubMed: 18854010]

7. Small KS, Brudno M, Hill MM, Sidow A. A haplome alignment and reference sequence of the highly polymorphic Ciona savignyi genome. Genome Biol. 2007; 8:R41. [PubMed: 17374142]

8. Vinson JP, Jaffe DB, O'Neill K, Karlsson EK, Stange-Thomann N, Anderson S, Mesirov JP, Satoh N, Satou Y, Nusbaum C, et al. Assembly of polymorphic genomes: algorithms and application to Ciona savignyi. Genome Res. 2005; 15:1127-1135. [PubMed: 16077012]

9. Hino K, Satou Y, Yagi K, Satoh N. A genomewide survey of developmentally relevant genes in Ciona intestinalis. VI. Genes for Wnt, TGFbeta, Hedgehog and JAK/STAT signaling pathways. Dev Genes Evol. 2003; 213:264-272. [PubMed: 12739142]

10. Sasakura Y, Shoguchi E, Takatori N, Wada S, Meinertzhagen IA, Satou Y, Satoh N. A genomewide survey of developmentally relevant genes in Ciona intestinalis. X. Genes for cell junctions and extracellular matrix. Dev Genes Evol. 2003; 213:303-313. [PubMed: 12740697]

11. Satou Y, Sasakura Y, Yamada L, Imai KS, Satoh N, Degnan B. A genomewide survey of developmentally relevant genes in Ciona intestinalis. V. Genes for receptor tyrosine kinase pathway and Notch signaling pathway. Dev Genes Evol. 2003; 213:254-263. [PubMed: 12739141]

12. Wada S, Tokuoka M, Shoguchi E, Kobayashi K, Di Gregorio A, Spagnuolo A, Branno M, Kohara Y, Rokhsar D, Levine M, et al. A genomewide survey of developmentally relevant genes in Ciona intestinalis. II. Genes for homeobox transcription factors. Dev Genes Evol. 2003; 213:222-234. [PubMed: 12736825]

13. Dehal P, Boore JL. Two rounds of whole genome duplication in the ancestral vertebrate. PLoS Biol. 2005; 3:e314. [PubMed: 16128622]

14. Hughes AL, Friedman R. Loss of ancestral genes in the genomic evolution of Ciona intestinalis. Evol Dev. 2005; 7:196-200. [PubMed: 15876192]

15. Chiba S, Jiang D, Satoh N, Smith WC. Brachyury null mutant-induced defects in juvenile ascidian endodermal organs. Development. 2009; 136:35-39. [PubMed: 19019990]

16. Jiang D, Munro EM, Smith WC. Ascidian prickle regulates both mediolateral and anteriorposterior cell polarity of notochord cells. Curr Biol. 2005; 15:79-85. [PubMed: 15700379]

17. Veeman MT, Nakatani Y, Hendrickson C, Ericson V, Lin C, Smith WC. Chongmague reveals an essential role for laminin-mediated boundary formation in chordate convergence and extension movements. Development. 2008; 135:33-41. [PubMed: 18032448]

18. Jiang D, Smith WC. Self-and cross-fertilization in the solitary ascidian Ciona savignyi. Biol Bull. 2005; 209:107-112. [PubMed: 16260770] 
19. Harada Y, Takagaki Y, Sunagawa M, Saito T, Yamada L, Taniguchi H, Shoguchi E, Sawada H. Mechanism of self-sterility in a hermaphroditic chordate. Science. 2008; 320:548-550. [PubMed: 18356489]

20. Hendrickson C, Christiaen L, Deschet K, Jiang D, Joly JS, Legendre L, Nakatani Y, Tresser J, Smith WC. Culture of adult ascidians and ascidian genetics. Methods Cell Biol. 2004; 74:143170. [PubMed: 15575606]

21. Small KS, Brudno M, Hill MM, Sidow A. Extreme genomic variation in a natural population. Proc Natl Acad Sci U S A. 2007; 104:5698-5703. [PubMed: 17372217]

22. Kim JH, Waterman MS, Li LM. Diploid genome reconstruction of Ciona intestinalis and comparative analysis with Ciona savignyi. Genome Res. 2007; 17:1101-1110. [PubMed: 17567986]

23. Shoguchi E, Kawashima T, Satou Y, Hamaguchi M, Sin IT, Kohara Y, Putnam N, Rokhsar DS, Satoh N. Chromosomal mapping of 170 BAC clones in the ascidian Ciona intestinalis. Genome Res. 2006; 16:297-303. [PubMed: 16354750]

24. Hill MM, Broman KW, Stupka E, Smith WC, Jiang D, Sidow A. The C. savignyi genetic map and its integration with the reference sequence facilitates insights into chordate genome evolution. Genome Res. 2008; 18:1369-1379. [PubMed: 18519652]

25. Satou Y, Takatori N, Fujiwara S, Nishikata T, Saiga H, Kusakabe T, Shin-i T, Kohara Y, Satoh N. Ciona intestinalis cDNA projects: expressed sequence tag analyses and gene expression profiles during embryogenesis. Gene. 2002; 287:83-96. [PubMed: 11992726]

26. Satou Y, Yamada L, Mochizuki Y, Takatori N, Kawashima T, Sasaki A, Hamaguchi M, Awazu S, Yagi K, Sasakura Y, et al. A cDNA resource from the basal chordate Ciona intestinalis. Genesis. 2002; 33:153-154. [PubMed: 12203911]

27. Imai KS, Hino K, Yagi K, Satoh N, Satou Y. Gene expression profiles of transcription factors and signaling molecules in the ascidian embryo: towards a comprehensive understanding of gene networks. Development. 2004; 131:4047-4058. [PubMed: 15269171]

28. Kusakabe T, Yoshida R, Kawakami I, Kusakabe R, Mochizuki Y, Yamada L, Shin-i T, Kohara Y, Satoh N, Tsuda M, et al. Gene expression profiles in tadpole larvae of Ciona intestinalis. Dev Biol. 2002; 242:188-203. [PubMed: 11820815]

29. Sasakura Y. Germline transgenesis and insertional mutagenesis in the ascidian Ciona intestinalis. Dev Dyn. 2007; 236:1758-1767. [PubMed: 17342755]

30. Deschet K, Nakatani Y, Smith WC. Generation of Ci-Brachyury-GFP stable transgenic lines in the ascidian Ciona savignyi. Genesis. 2003; 35:248-259. [PubMed: 12717736]

31. Zeller RW, Virata MJ, Cone AC. Predictable mosaic transgene expression in ascidian embryos produced with a simple electroporation device. Dev Dyn. 2006; 235:1921-1932. [PubMed: 16607640]

32. Nydam ML, Harrison RG. Genealogical relationships within and among shallow-water Ciona species (Ascidiacea). Mar Biol. 2007; 151:1839-1847.

33. Hoshino Z, Nishikawa T. Taxonomic studies of Ciona intestinalis (L.) and its allies. Publ Seto Mar Biol Lab. 1985; 30:61-79.

34. Silva N, Smith WC. Inverse correlation of population similarity and introduction date for invasive ascidians. PLoS One. 2008; 3:e2552. [PubMed: 18575620]

35. Dybern BI. The life cycle of Ciona intestinalis (L.) f. typica in relation to the environmental temperature. Oikos. 1965; 16:109-131.

36. Joly JS, Kano S, Matsuoka T, Auger H, Hirayama K, Satoh N, Awazu S, Legendre L, Sasakura Y. Culture of Ciona intestinalis in closed systems. Dev Dyn. 2007; 236:1832-1840. [PubMed: 17394236]

37. Suzuki MM, Nishikawa T, Bird A. Genomic approaches reveal unexpected genetic divergence within Ciona intestinalis. J Mol Evol. 2005; 61:627-635. [PubMed: 16205978] 

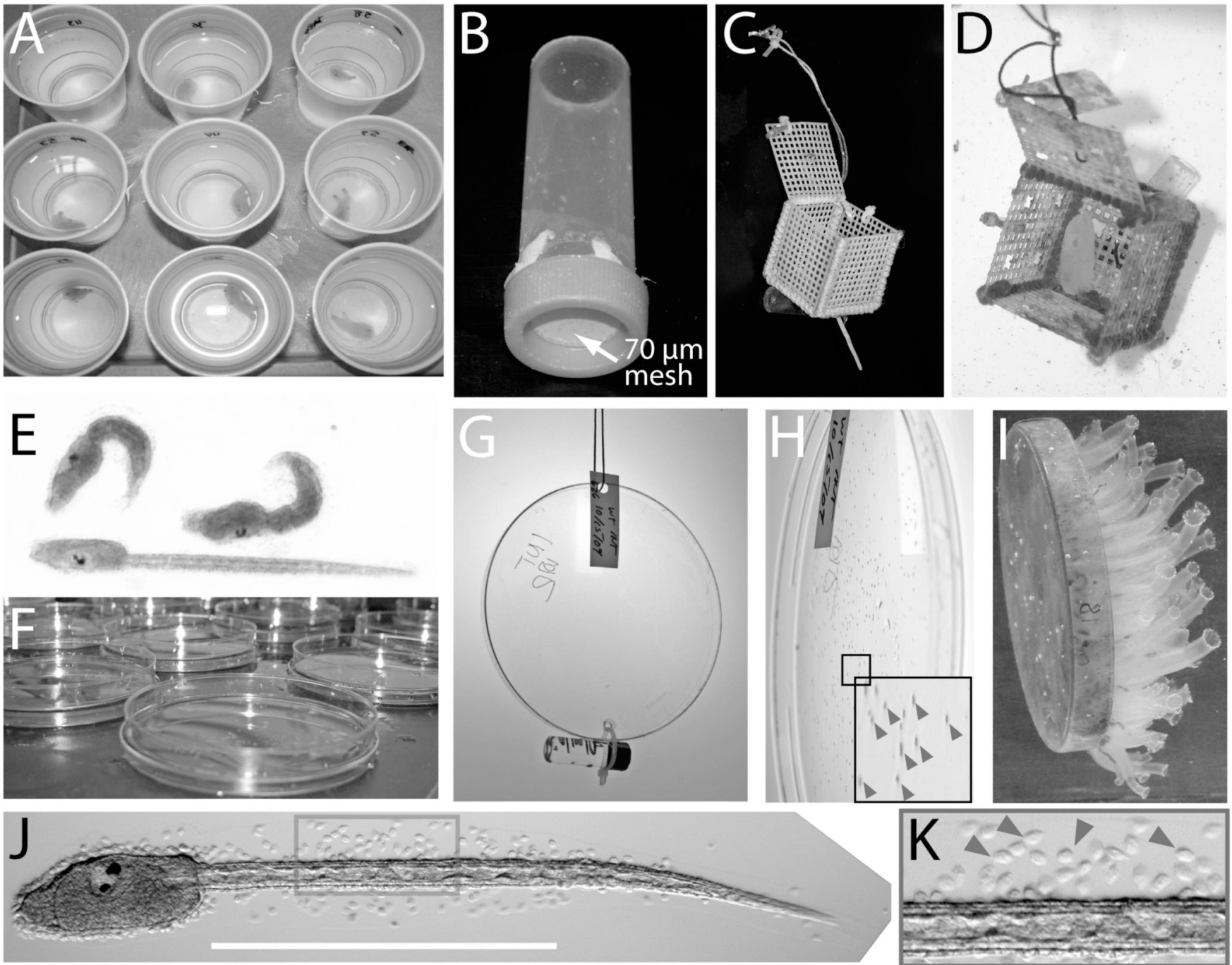

Fig. 1. Tools and techniques for ascidian genetics

A) Disposable plastic drink cups make ideal vessels for spawning single ascidians.

B) Homemade egg filters with a $70 \mu \mathrm{m}$ nylon mesh bottom are indispensable for washing eggs and embryos, and for performing certain types of crosses.

C) 'Condos' are used to house and label single ascidians after they have been detached from their original substrate.

D) An opened condo containing an attached adult.

E) An example of a wildtype larva (bottom) together with two mutant siblings.

F) Minimize the air-water interface when settling larvae onto Petri dishes by using the lid (concave side up) as a dish to hold swimming larvae, and then float the part that is normally used as the dish on top (also concave side up).

G) Prepare a plate with attached juveniles for long-term culture by making two holes on opposite sides of the plate with a soldering iron. Attach a small weight, such as a scintillation vial to one hole with a zip tie, and tie a loop of string through the other hole. H) Attachment density can be checked by briefly pouring off the water and viewing the plate at an angle. Juveniles are easily seen in oblique light as small bumps on the plate (indicated with arrowheads in inset).

I) A plate of adult $C$. savignyi. 
J) A hatched, swimming larva of $C$. intestinalis. Scale bar is $0.5 \mathrm{~mm}$. Green box indicates the closeup in $\mathrm{K}$.

K) Closeup view of a segment of tail showing numerous test cells (some indicated with arrowheads) in the larval tunic. 

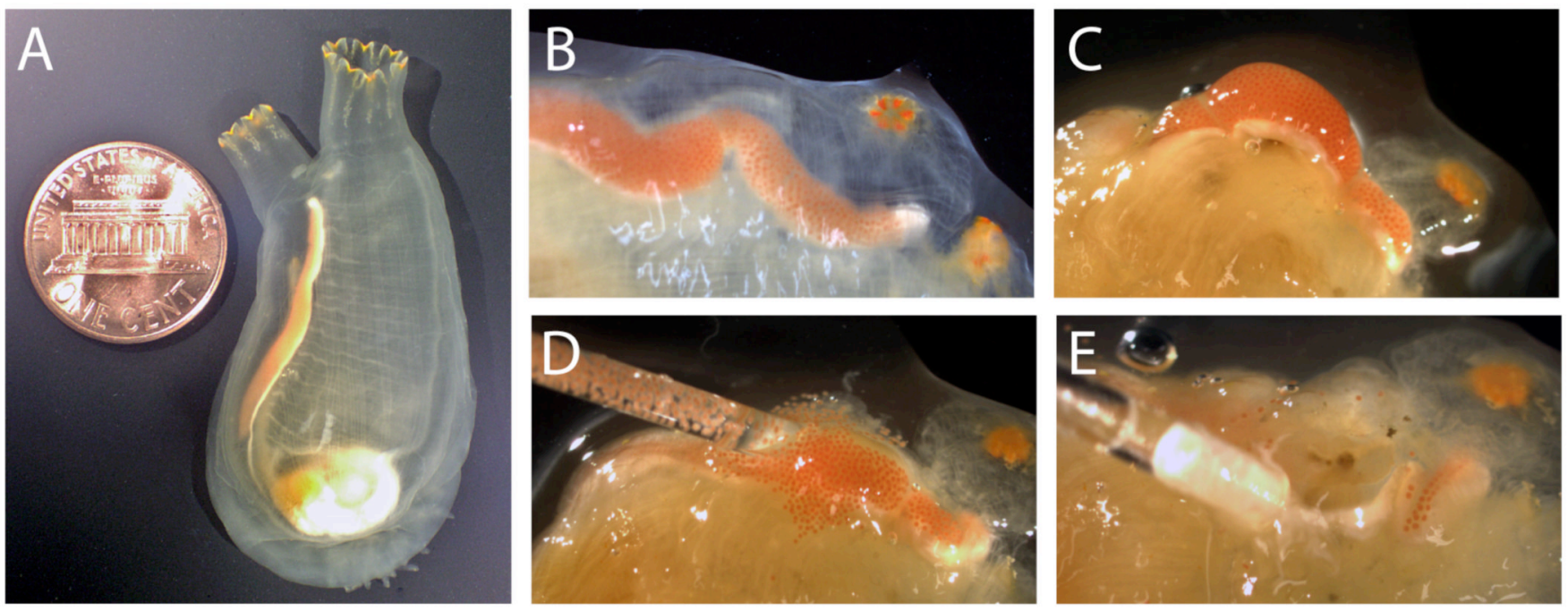

Fig. 2. Collecting gametes by dissection

A) Gravid adult Ciona savignyi. Note the parallel ooduct (orange) and sperm duct (white).

B) Closeup of ooduct and sperm duct after the tunic has been removed.

C) The body wall muscle layer has been dissected open to expose the ooduct and sperm duct.

D) The ooduct has been pierced and the eggs are being collected with a Pasteur pipette.

E) The sperm duct has been pierced and sperm is being collected with a Pasteur pipette. 


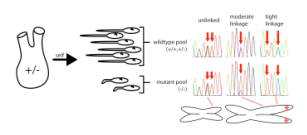

Fig. 3. Mapping by direct sequencing of SNPs

Initial linkage can be detected by preparing pools of DNA from phenotypically wildtype versus mutant individuals from a single selfed heterozygote. Use the two pools as templates for PCR reactions using primer pairs evenly spaced through the genome. SNPs can be detected as double peaks in the sequencing chromatogram for the wildtype pool. The ratio of these peak heights is repeatable for a given pool but varies considerably from SNP to SNP through sequence specific variation in polymerase incorporation efficiency. Linkage can be detected as a variation in the relative peak heights in the mutant pool versus the wildtype pool. This becomes more extreme with markers increasingly close to the mutant locus (indicated with a red asterisk). 

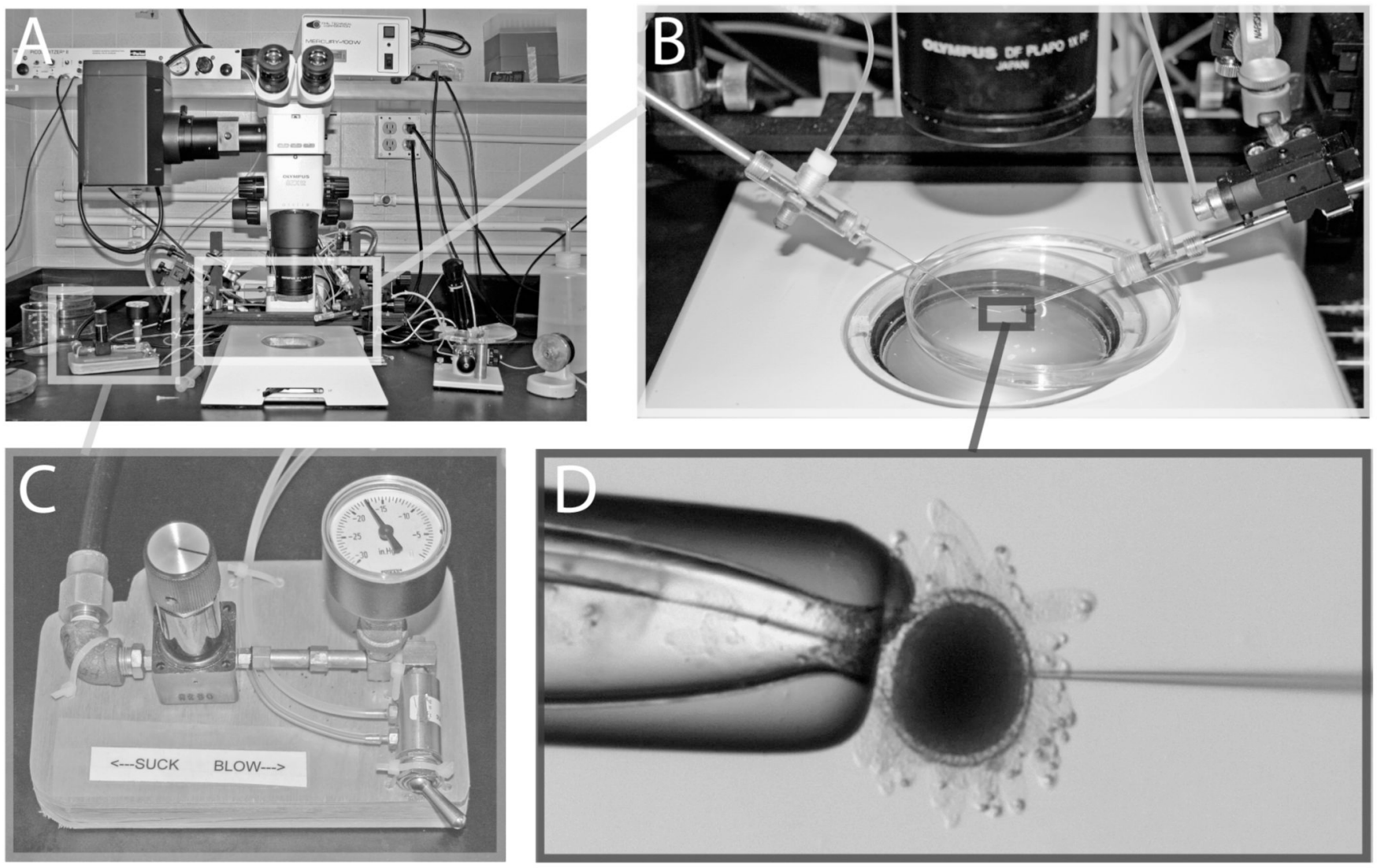

Fig. 4. Microinjection of $C$. savignyi eggs

A) Overview of injection station.

B) Close up showing the holding pipette (left) and injection pipette (right)

C) Close up of the homemade vacuum/pressure switching device.

D) High-magnification view of injection, showing the relative proportions of the egg (surrounded by the chorion and an outer layer of follicle cells), the holding pipette and the injection pipette. 